\title{
Intrathoracic Migration of a Steinman Pin after Glenohumeral Fixation-The Journey Continues
}

\author{
Ellen Deleus ${ }^{1}$, Tine Grégoir ${ }^{2}$, Boris Robic ${ }^{1,3}$, Urbain Mees ${ }^{1}$, Carl Dierickx ${ }^{2,3}$, Gerrit De Wachter ${ }^{2}$, \\ Marc Hendrikx ${ }^{1,3}$ \\ ${ }^{1}$ Department of Cardiothoracic Surgery, Jessa Hospital, Hasselt, Belgium; ${ }^{2}$ Department of Orthopaedic Surgery, Jessa Hospital, \\ Hasselt, Belgium; ${ }^{3}$ Faculty of Medicine and Life Sciences, Hasselt University, Hasselt, Belgium. \\ Email: marc.hendrikx@jessazh.be
}

Received June $14^{\text {th }}, 2013$; revised July $13^{\text {th }}, 2013$; accepted July $27^{\text {th }}, 2013$

Copyright (C 2013 Ellen Deleus et al. This is an open access article distributed under the Creative Commons Attribution License, which permits unrestricted use, distribution, and reproduction in any medium, provided the original work is properly cited.

\begin{abstract}
We report the case of a 71-year-old woman, who was treated for intrathoracic migration of a Steinman pin, after glenohumeral fixation of an instable dislocation. A thoracotomy was necessary to retrieve the pin. We believe this case can be a reminder of the risks of transarticular fixation around the shoulder. A review of the literature shows very little information regarding glenohumeral pinning.
\end{abstract}

Keywords: Glenohumeral Dislocation; Transarticular Fixation; Migration of Osteosynthetic Material

\section{Introduction}

Instable shoulder dislocation in patients with repetitive falling can be a challenging problem for orthopedic surgeons. However, reports about glenohumeral fixation using percutaneous wires or pins are scarce and inconclusive. With this case report we would like to remind to be cautious when performing this technique.

\section{Case Report}

A 71-year-old female patient was admitted to our hospital on referral of her general practitioner because of psychological decompensation and repetitive falling. She had previously been diagnosed with a depressive disorder. The repetitive falling had been attributed to a conversion disorder (DSM-IV-TR 300.11).

She presented with a painful right shoulder, on clinical examination an extensive hematoma reaching from the right shoulder to the ipsilateral hip was seen. Mobilization of the right shoulder was impossible due to pain. A complete tear of the supraspinatus tendon was diagnosed. $\mathrm{X}$-ray of the shoulder revealed a normal articulation. She was discharged home. Two days later the patient was readmitted because of increasing pain and loss of function of the right shoulder. She reported no trauma. X-rays revealed an anterior shoulder dislocation, with an associated bony Bankart lesion.
A closed reduction of the dislocation turned out to be impossible and she was treated with an open reduction. There was an important intraoperative instability of the shoulder, therefore a smooth Steinman pin was placed percutaneously, through the humeral head and the glenoid. This was a temporary stabilization, with the intention to perform a total shoulder replacement later. A sterile draping was applied. Imaging with anteroposterior Figure 1, and lateral X-rays confirmed good position of the shoulder and the pin. Because of her general condition the patient was admitted to the orthopedic ward. She continued to complain of severe pain in both shoulders. Two weeks postoperatively, the tip of the Steinman pin was no longer visualized externally. An urgent X-ray was taken, on which an important migration of the Steinman pin, medially, towards the thoracic vertebrae was seen Figure 2.

The patient was referred to the department of cardiothoracic surgery. A CT-scan was performed to map the intracorporeal course of the pin Figure 3.

The pin was displaced through the apex of the right lung, reaching dorsomedially into the spinal canal of T3/4, immediately behind the dural sac. A fractured second rib and a small hemopneumothorax were also seen.

The patient was taken to the operating theatre the next day. A right posterior thoracotomy through the forth intercostal space was performed. On opening the thorax we 


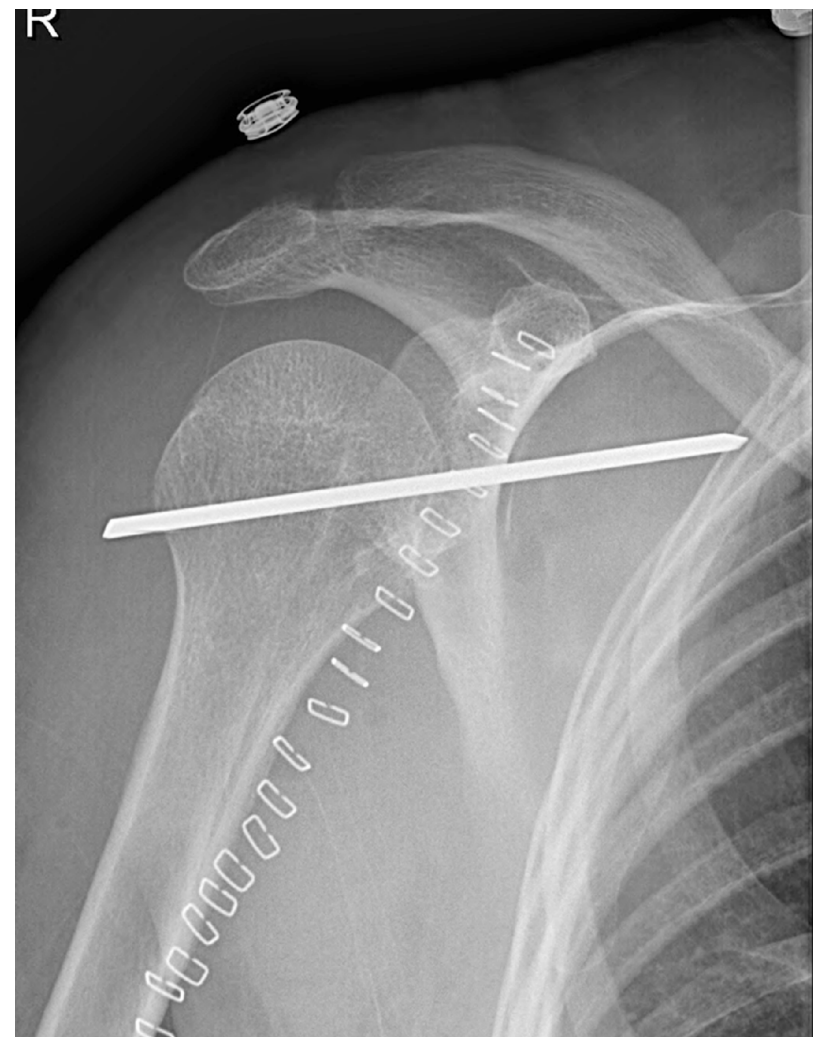

Figure 1. Anteroposterior X-Ray showing correct positioning of the glenohumeral pinning.

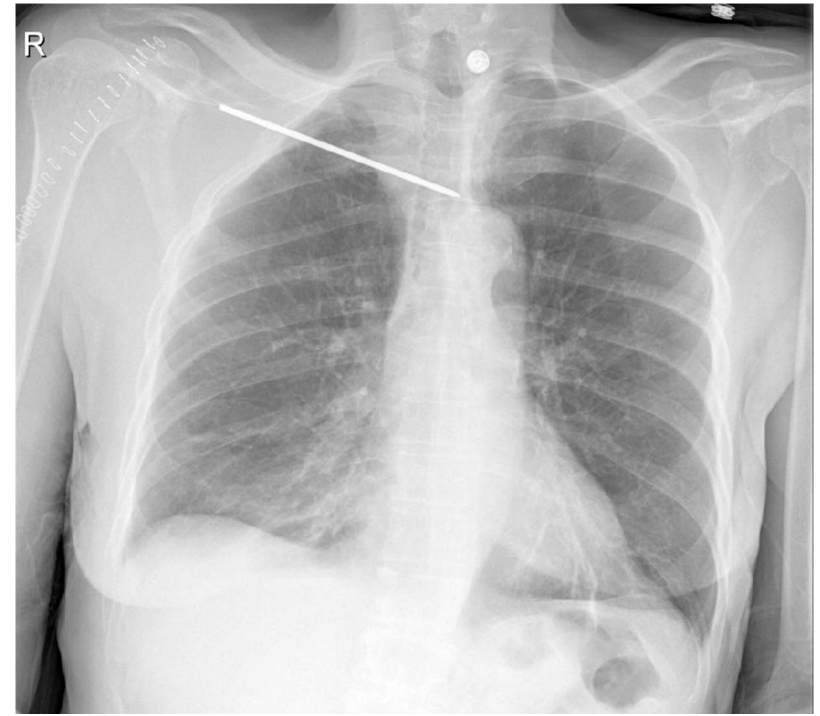

Figure 2. Urgent X-Ray, showing important medial migration of the pin.

clearly visualized the metal pin, which was piercing the apex of the right lung Figure 4.

The pin was removed by pushing it firmly back towards the shoulder and then taking it out, using a heavy needle-holder. The patient was extubated in the operating room; she showed no signs of neurological deficit. The

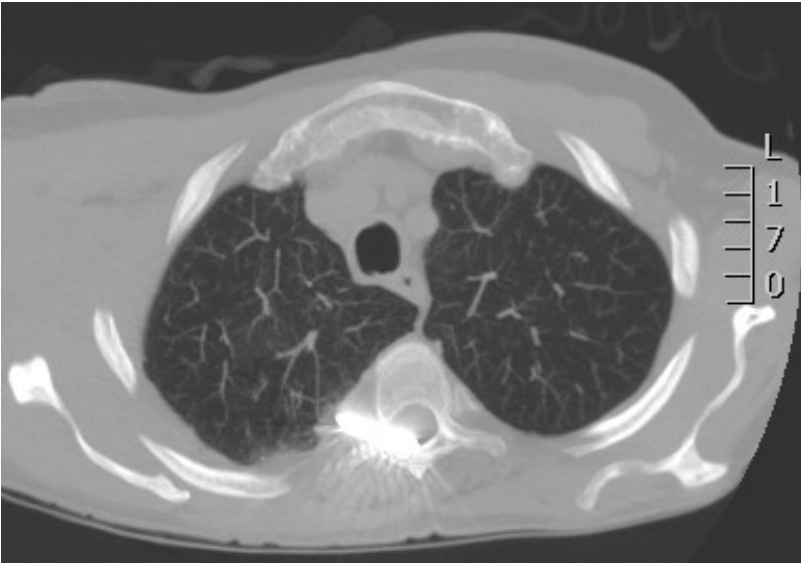

Figure 3. CT-imaging: migration of the pin through the apex of the right lung, towards the spinal canal Th3/Th4.

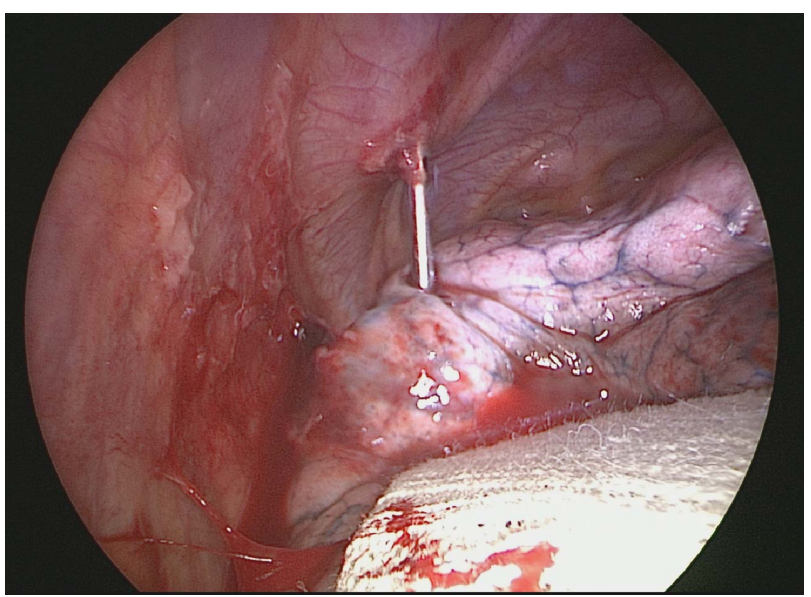

Figure 4. Intraoperative view during right posterior thoracotomy. Steinman pin piercing the apex of the right lung.

postoperative course was uneventful; the shoulder was further immobilized in a sling. X-rays 11 days postoperatively showed a normal shoulder articulation. After her stay in the surgical department, she was transferred to the geriatric ward for further recovery. Eventually she was admitted to a psychiatric hospital due to persistent deliberate falling with multiple facial lacerations. The patient presented for a follow-up visit 7 months later with good shoulder function. The glenohumeral articulation had remained stable and X-rays showed correct position of the joint.

\section{Discussion}

Open reduction of a dislocated shoulder can be complicated by severe instability of the shoulder; glenohumeral pinning has been described as a valid temporary treatment option for an instable shoulder, as suggested by Neviaser [1].

As described by Lyons et al. [2] in 1990, pins and 
wires around the shoulder should be used with extreme caution and precaution measures, such as using a threaded pin, bending the external end of the pin and choosing a correct diameter of the pin. We conducted a literature search to investigate the use of glenohumeral pinning and occurrence of pin or wire migration. Search terms include: "Glenohumeral fixation", "Glenohumeral transarticular fixation", "Glenohumeral pinning”. It became clear that this method is applied in very specific and rare cases. Reports about migration with disastrous results are even more infrequent.

Mazet [3] first reported Kirschner-wire migration from the shoulder joint towards the lung in 1943. The second patient he described was a 16-year-old girl who presented with a right shoulder flail after suffering from poliomyelitis. Three K-wires were placed through the humeral head and into the glenoid. Sixteen weeks after the operation one of the wires was found to be protruding both lungs, the wire was removed successfully.

Was et al. [4] published the case of a 66-year-old man with an inveterate anterior shoulder dislocation. A smooth $\mathrm{K}$-wire was placed through the glenohumeral articulation. The shoulder was immobilized in a sling. A routine follow-up on day 26 postoperatively showed an asymptomatic migration into the right thoracic cavity reaching trough the right Th8 nerve root foramen. The pin was retrieved by posterolateral thoracotomy. The patient made a good recovery.

Potter et al. [5] reported the case of a 79-year-old woman with an unstable posterior glenohumeral luxation. Two 13-cm-long Kirschner wires were inserted through the humeral head into the glenoid. Five days later one wire was found to be intrathoracic, the patient was however not assumed to be fit for surgery. After two days the wire was removed during laparotomy with subsequent splenectomy. She recovered well.

A fourth case was described by Sarper et al. [6]: a 55-year-old women sustained a shoulder dislocation, which was treated with open reduction and fixation by means of a Steinman pin. One month after surgery she was re-admitted with severe back pain, the migrated wire was removed during thoracotomy.

There is only very little information in the literature regarding successful temporary glenohumeral pinning to treat instable shoulder luxation. Vandeputte and Van Tornout [7] published a series of 4 patients with severely instable posterior shoulder dislocation. All four patients were treated with closed reduction and percutaneous pinning. Glenohumeral pinning was achieved with 3 to 4 diverging threaded $2.5 \mathrm{~mm}$ pins, the pin ends were bowed. In one case the pins were removed after four instead of six weeks, because of asymptomatic intrathoracic migration of one of the pins, this pin had not been bent to $90^{\circ}$. In all other cases the pins remained correctly positioned, they were removed after 6 weeks.

Widmaier and Fanelli [8] describe the case of a 93year-old patient. She was treated for recurrent instability following an anterior glenohumeral dislocation with associated glenoid fracture after a fall. A percutaneous approach was chosen in this patient, because of previous irradiation leaving her with contracted tissues around the axilla. Two smooth 0.62 inch Kirschner wires were placed from the great tuberosity into the glenoid. It is unclear if the pins were bowed at the end. A shoulder immobilizer was used to protect the stabilization. Interestingly, this lady fell again on the same side two weeks later, sustaining an ipsilateral femoral neck fracture. The glenohumeral reduction remained. At 7 weeks postoperatively the pins were removed, she made a good recovery, with no limitations in her daily activities. The authors advocate to reserve this technique for a select group of patients with recurrent instability due to an anterior glenohumeral dislocation, and in whom open surgery is not an option.

\section{Conclusion}

Glenohumeral pinning is used in exceptional cases of shoulder instability. However, based on the limited information in literature, there is a considerable risk of migration. Even with careful follow-up and precautious measures such as bending the ends of the pins and wires, migration of pins and wires around the shoulder has been described. Other treatment options for difficult cases of shoulder instability include conservative approach by simply supporting the arm, hemiarthroplasty or a total shoulder replacement. The decision should be based on radiological findings, as well as the age and operative risk of the patient.

\section{REFERENCES}

[1] T. J. Neviaser, "Old Unreduced Dislocations of the Shoulder," Orthopedic Clinics of North America, Vol. 11, No. 2, 1980, pp. 287-294.

[2] F. A. Lyons and C. A. Rockwood, "Migration of Pins Used in Operations on the Shoulder," Journal of Bone and Joint Surgery, Vol. 72, No. 8, 1990, pp. 1262-1267.

[3] R. Mazet, "Migration of a Kirschner Wire from the Shoulder Region into the Lung: Report of Two Cases," Journal of Bone and Joint Surgery, Vol. 25, No. 2, 1943, pp. 477-483.

[4] M. T. Was, K. Kurowski and I. Francuz, "Migration of Kirschner Wire into the Spinal Canal as c-Complication of Inveterate Shoulder Luxation Treatment-Case Study," Ortopedia Traumatologia Rehabilitacja, Vol. 12, No. 4, 2010, pp. 370- 275.

[5] F. A. Potter, A. J. Fiorini, J. Knox, et al., "The Migration of a Kirschner Wire from Shoulder to Spleen: Brief Report,” Journal of Bone and Joint Surgery, Vol. 70, No. 2, 1988, pp. 326-327. 
[6] A. Sarper, M. Ürgüden, L. Dertsiz, et al., "Intrathoracic Migration of Steinman Wire," Interactive Cardio Vascular and Thoracic Surgery, Vol. 2, No. 2, 2003, pp. 210211. doi:10.1016/S1569-9293(03)00047-1

[7] J. Vandeputte and B. Van Tornout, "Missed Posterior Fracture Dislocation of the Shoulder: Closed Reduction and Pinning," Acta Orthopaedica Belgica, Vol. 59, No. 2,
1993, pp. 225-230.

[8] J. C. Widmaier and G. Fanelli, “Temporary Transarticular Stabilization of a Glenohumeral Fracture Dislocation: A Technique Revisited,” Journal of Orthopaedic Trauma, Vol. 17, No. 9, 2003, pp. 644-647. doi:10.1097/00005131-200310000-00008 